\title{
Conjugated Polymers Containing Large Soluble Diethynyl Iptycenes
}

\author{
Supporting Information
}

Dahui Zhao and Timothy M. Swager*

Department of Chemistry, Massachusetts Institute of Technology, Cambridge, Massachusetts 02139.

tswager@mit.edu

General Methods and Materials: ${ }^{1} \mathrm{H}$ and ${ }^{13} \mathrm{C}$ NMR spectra were recorded on a Varian Mercury-300 MHz or a Varian Unity-500 MHz instrument. Chemical shifts are reported in parts per million relative to tetramethylsilane or $\mathrm{CHCl}_{3}$. Mass spectra were obtained on a Bruker Daltonics APEXII3 Tesla FTICR mass spectrometer using electrospray ionization. Polymer molecular weights were determined by gel permeation chromatography using an HP series 1100 GPC system running at a flow rate at 1.0 $\mathrm{mL} / \mathrm{min}$ in THF and a diode detector. The molecular weights are reported relative to the polystyrene standards purchased from Polysciences. Anhydrous N,N-dimethylformamide (DMF), toluene and diisopropylamine (DIPA) were purchased from Aldrich. 6,13-Bis(triisopropylsilylethynyl)pentacene ${ }^{1}$ and triptycenequinone ${ }^{2}$ were synthesized as previously reported. All other solvents and compounds are purchased from Aldrich or Lancaster and used as received unless otherwise noted. Silica gel and thin layer chromatography plates were purchased from J. T. Baker. All air- and moisture-sensitive synthetic procedures were performed under argon atmosphere. 


\section{Syntheses}

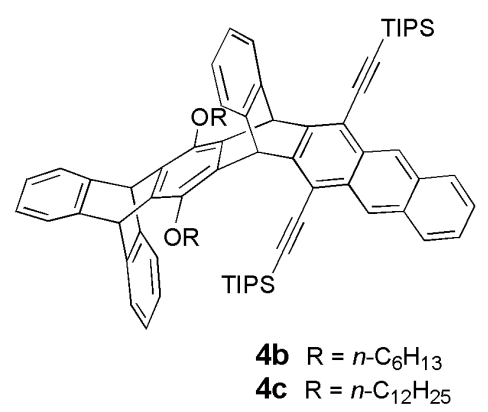

A mixture of 6,13-bis(triisopropylsilylethynyl)pentacene (2.66 g, $4.2 \mathrm{mmol})$ and triptycenequinone $(1.42 \mathrm{~g}, 5.0 \mathrm{mmol})$ was dissolved in toluene $(35 \mathrm{~mL})$. The reaction vessel was then sealed, heated at 110 ${ }^{\circ} \mathrm{C}$ with stirring for 3 hours and then cooled back to rt. The solvent was removed in vacuo. The crude product was purified by silica gel column chromatography eluting with $\mathrm{CH}_{2} \mathrm{Cl}_{2} / \mathrm{Hexanes}(2: 3$, v/v) to give yellow crystals as the pure product $\left(3.2 \mathrm{~g}\right.$, identified by ${ }^{1} \mathrm{H}$ NMR as one of the two possible isomers). The yellow crystals were then transferred into a Schlenk flask along with sodium hydride $(0.20 \mathrm{~g}, 8.3 \mathrm{mmol})$ and potassium iodide $(0.17 \mathrm{~g}, 1.0 \mathrm{mmol})$. The flask was evacuated and back-filled with argon three times. Under argon atmosphere, anhydrous DMF (45 mL) was added. After stirring the mixture at $\mathrm{rt}$ for $5 \mathrm{~min}, n$-dodecylbromide $(2.5 \mathrm{~mL}, 10.0 \mathrm{mmol})$ was added dropwise. The flask was then sealed and heated at $60{ }^{\circ} \mathrm{C}$ overnight. The reaction mixture was cooled to $\mathrm{rt}$ and diluted with methylene chloride. The solution was washed with saturated $\mathrm{NH}_{4} \mathrm{Cl}$ (aq.) twice and followed by brine twice. The organic layer was dried over $\mathrm{Na}_{2} \mathrm{SO}_{4}$ and concentrated in vacuo. The crude product was purified by column chromatography eluting with $\mathrm{CH}_{2} \mathrm{Cl}_{2} / \mathrm{Hexanes}(1: 4, \mathrm{v} / \mathrm{v})$ to give $\mathbf{4 c}$ as light green oil (3.9 g, 75\%), which slowly turned into tacky solid when stored in the refrigerator. $4 \mathbf{b}$ was synthesized using the same procedure except $n$-hexyliodide was used in the place of $n$-dodecylbromide and KI. $\mathbf{4 b}$ : ${ }^{1} \mathrm{H}$ NMR (500 MHz, $\left.\mathrm{CDCl}_{3}\right) 8.88(\mathrm{~s}, 2 \mathrm{H}), 7.97(\mathrm{dd}, 2 \mathrm{H}), 7.48(\mathrm{dd}, 2 \mathrm{H}), 7.42(\mathrm{~m}, 2 \mathrm{H}), 7.38(\mathrm{~m}, 4 \mathrm{H}), 7.07$ (dd, 2H), $7.00(\mathrm{dd}, 2 \mathrm{H}), 6.95(\mathrm{dd}, 2 \mathrm{H}), 6.48(\mathrm{~s}, 2 \mathrm{H}), 5.79(\mathrm{~s}, 2 \mathrm{H}), 4.21(\mathrm{~m}, 2 \mathrm{H}), 4.02(\mathrm{~m}, 2 \mathrm{H}), 2.05(\mathrm{~m}$, 4H), $1.62(\mathrm{~m}, 4 \mathrm{H}), 1.43(\mathrm{~m}, 50 \mathrm{H}), 1.03(\mathrm{t}, 6 \mathrm{H}) .{ }^{13} \mathrm{C} \mathrm{NMR}\left(125 \mathrm{MHz}, \mathrm{CDCl}_{3}\right)$ 146.6, 145.6, 145.4, 144.4, 144.1, 137.2, 134.7, 132.2, 129.6, 128.5, 126.0, 125.82, 125.79, 125.3, 125.2, 124.2, 123,8, 123.7, 115,8, 
103.0, 101.6, 76.7, 48.6, 47.1, 32.1, 30.8, 26.1, 23.0, 19.23, 19.19, 14.4, 11.8. HRMS calcd for $\mathrm{C}_{76} \mathrm{H}_{91} \mathrm{O}_{2} \mathrm{Si}_{2}[\mathrm{M}+\mathrm{H}]^{+}$1091.6552, found 1091.6552. 4c: ${ }^{1} \mathrm{H}$ NMR (500 MHz, $\left.\mathrm{CDCl}_{3}\right) 8.81(\mathrm{~s}, 2 \mathrm{H}), 7.91$ (dd, 2H), $7.43(\mathrm{dd}, 2 \mathrm{H}), 7.36(\mathrm{~m}, 2 \mathrm{H}), 7.31(\mathrm{~m}, 4 \mathrm{H}), 7.00(\mathrm{dd}, 2 \mathrm{H}), 6.94(\mathrm{dd}, 2 \mathrm{H}), 6.89(\mathrm{dd}, 2 \mathrm{H}), 6.41(\mathrm{~s}$, 2H), $5.71(\mathrm{~s}, 2 \mathrm{H}), 4.13(\mathrm{~m}, 2 \mathrm{H}), 3.95(\mathrm{~m}, 2 \mathrm{H}), 1.98(\mathrm{~m}, 4 \mathrm{H}), 1.55(\mathrm{~m}, 4 \mathrm{H}), 1.32(\mathrm{~m}, 74 \mathrm{H}), 0.89(\mathrm{t}, 6 \mathrm{H})$. ${ }^{13} \mathrm{C}$ NMR (125 MHz, $\left.\mathrm{CDCl}_{3}\right)$ 146.6, 145.7, 145.4, 144.4, 144.1, 137.2, 134.7, 132.2, 129.7, 128.5, 126.1, $125.84,125.82,125.3,125.2,124.2,123.9,123.8,115,8,103.0,101.6,76.8,48.7,47.1,32.2,30.8$, $30.02,30.00,29.97,29.93,29.6,26.5,23.0,19.26,19.23,14.4,11.9$. HRMS calcd for $\mathrm{C}_{88} \mathrm{H}_{114} \mathrm{O}_{2} \mathrm{Si}_{2} \mathrm{Na}$ $[\mathrm{M}+\mathrm{Na}]^{+} 1281.8250$, found 1281.8178 .
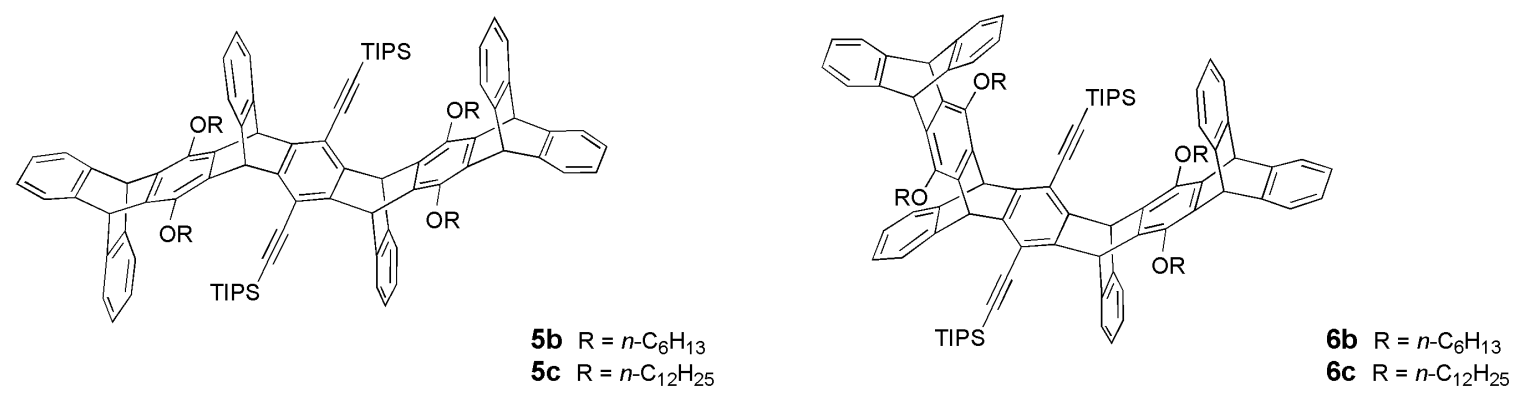

A mixture of $4 \mathbf{c}(3.7 \mathrm{~g}, 2.9 \mathrm{mmol})$ and triptycene quinone $(1.7 \mathrm{~g}, 5.8 \mathrm{mmol})$ was dissolved in xylenes $(40 \mathrm{~mL})$. The reaction vessel was then sealed, heated at $140{ }^{\circ} \mathrm{C}$ with stirring for 2 days and then cooled back to rt. The solvent was removed in vacuo. The crude product was applied to silica gel column chromatography and eluted with $\mathrm{CH}_{2} \mathrm{Cl}_{2} / \mathrm{Hexanes}(2: 3, \mathrm{v} / \mathrm{v})$ to give the two isomeric products (the reaction was incomplete and some starting materials were recovered). The two isomeric products were combined and the mixture ( $3.3 \mathrm{~g})$ was transferred into a Schlenk flask along with sodium hydride ( 0.13 $\mathrm{g}, 5.4 \mathrm{mmol})$ and potassium iodide $(0.11 \mathrm{~g}, 0.67 \mathrm{mmol})$. The flask was evacuated and back-filled with argon three times. Under argon atmosphere, anhydrous DMF $(40 \mathrm{~mL})$ was added. After stirring the mixture at $\mathrm{rt}$ for $5 \mathrm{~min}, n$-dodecylbromide $(1.6 \mathrm{~mL}, 6.7 \mathrm{mmol})$ was added dropwise. The flask was then sealed and heated at $60{ }^{\circ} \mathrm{C}$ overnight. The reaction mixture was cooled to rt and diluted with methylene chloride. The solution was washed with saturated $\mathrm{NH}_{4} \mathrm{Cl}$ (aq.) twice and followed by brine twice. The 
organic layer was dried over $\mathrm{Na}_{2} \mathrm{SO}_{4}$ and concentrated in vacuo. The crude product was purified by column chromatography eluting with $\mathrm{CH}_{2} \mathrm{Cl}_{2} / \mathrm{Hexanes}(1: 3$, v/v) to give 5c first $(1.8 \mathrm{~g}, 33 \%)$, and followed by $\mathbf{6 c}(1.7 \mathrm{~g}, 31 \%)$. Both products were sticky solids. $5 \mathbf{b}$ and $\mathbf{6 b}$ was synthesized using the same procedure except $n$-hexyliodide was used in the place of $n$-dodecylbromide and KI. $5 \mathbf{b}$ : ${ }^{1} \mathrm{H}$ NMR $\left(500 \mathrm{MHz}, \mathrm{CDCl}_{3}\right) 7.28(\mathrm{~m}, 8 \mathrm{H}), 7.17(\mathrm{~m}, 4 \mathrm{H}), 6.94(\mathrm{~m}, 4 \mathrm{H}), 6.87(\mathrm{~m}, 4 \mathrm{H}), 6.83(\mathrm{~m}, 4 \mathrm{H}), 6.12(\mathrm{~s}, 4 \mathrm{H})$, $5.65(\mathrm{~s}, 4 \mathrm{H}), 3.91(\mathrm{~m}, 8 \mathrm{H}), 1.91(\mathrm{~m}, 8 \mathrm{H}), 1.43(\mathrm{~m}, 66 \mathrm{H}), 0.99(\mathrm{t}, 12 \mathrm{H}) .{ }^{13} \mathrm{C} \mathrm{NMR}\left(125 \mathrm{MHz}, \mathrm{CDCl}_{3}\right)$ $146.5,145.9,145.7,145.5,144.5,136.4,135.9,125.14,125.12,125.07,123.8,123.64,123.59,115.2$, 102.4, 98.8, 76.4, 48.6, 46.9, 32.1, 30.6, 26.0, 23.0, 19.3, 19.2, 14.4, 12.0. HRMS calcd for $\mathrm{C}_{108} \mathrm{H}_{126} \mathrm{O}_{4} \mathrm{Si}_{2} \mathrm{Na}[\mathrm{M}+\mathrm{Na}]^{+}$1565.9087, found $1565.9022 .6 \mathbf{b}:{ }^{1} \mathrm{H} \mathrm{NMR}\left(500 \mathrm{MHz}, \mathrm{CDCl}_{3}\right) 7.27(\mathrm{~m}, 4 \mathrm{H})$, $7.23(\mathrm{~m}, 4 \mathrm{H}), 7.19(\mathrm{~m}, 4 \mathrm{H}), 6.91(\mathrm{~m}, 4 \mathrm{H}), 6.86(\mathrm{~m}, 8 \mathrm{H}), 6.12(\mathrm{~s}, 4 \mathrm{H}), 5.61(\mathrm{~s}, 4 \mathrm{H}), 4.03(\mathrm{~m}, 4 \mathrm{H}), 3.76$ (m, 4H) $1.92(\mathrm{~m}, 8 \mathrm{H}), 1.54(\mathrm{~m}, 8 \mathrm{H}), 1.40(\mathrm{~m}, 58 \mathrm{H}), 1.01(\mathrm{t}, 12 \mathrm{H}) .{ }^{13} \mathrm{C}$ NMR $\left(125 \mathrm{MHz}, \mathrm{CDCl}_{3}\right)$ 146.4, 145.64, 145.58, 145.52, 144.6, 136.4, 135.8, 125.3, 125.2, 125.0, 123.71, 123.68, 123.62, 115.3, 102.7, 98.8, 76.5, 48.5, 46.8, 32.2, 30.8, 26.2, 23.0, 19.3, 14.4, 11.8. HRMS calcd for $\mathrm{C}_{108} \mathrm{H}_{126} \mathrm{O}_{4} \mathrm{Si}_{2} \mathrm{Na}$ $[\mathrm{M}+\mathrm{Na}]^{+}$1565.9087, found 1565.9115. 5c: ${ }^{1} \mathrm{H}$ NMR (500 MHz, $\left.\mathrm{CDCl}_{3}\right) 7.26(\mathrm{~m}, 4 \mathrm{H}), 7.25(\mathrm{~m}, 4 \mathrm{H})$, $7.14(\mathrm{~m}, 4 \mathrm{H}), 6.91(\mathrm{~m}, 4 \mathrm{H}), 6.85(\mathrm{~m}, 4 \mathrm{H}), 6.80(\mathrm{~m}, 4 \mathrm{H}), 6.09(\mathrm{~s}, 4 \mathrm{H}), 5.62(\mathrm{~s}, 4 \mathrm{H}), 3.87(\mathrm{~m}, 8 \mathrm{H}), 1.87$ (m, 8H), $1.34(\mathrm{~m}, 114 \mathrm{H}), 0.90(\mathrm{t}, 12 \mathrm{H}) .{ }^{13} \mathrm{C} \mathrm{NMR}\left(125 \mathrm{MHz}, \mathrm{CDCl}_{3}\right)$ 146.5, 145.8, 145.7, 145.4, 144.5, $136.4,135.8,125.10,125.08,125.03,123.7,123.6,123.5,115.2,102.3,98.7,76.4,48.5,46.9,32.1$, 30.7, 29.98, 29.92, 29.89, 29.6, 26.4, 22.9, 19.3, 19.2, 14.4, 12.0. HRMS calcd for $\mathrm{C}_{132} \mathrm{H}_{174} \mathrm{O}_{4} \mathrm{Si}_{2} \mathrm{Na}$ $[\mathrm{M}+\mathrm{Na}]^{+}$1902.2843, found 1902.2849. 6c: ${ }^{1} \mathrm{H}$ NMR (500 MHz, $\left.\mathrm{CDCl}_{3}\right) 7.26(\mathrm{~m}, 4 \mathrm{H}), 7.22(\mathrm{~m}, 4 \mathrm{H})$, $7.18(\mathrm{~m}, 4 \mathrm{H}), 6.90(\mathrm{~m}, 4 \mathrm{H}), 6.84(\mathrm{~m}, 8 \mathrm{H}), 6.11(\mathrm{~s}, 4 \mathrm{H}), 5.60(\mathrm{~s}, 4 \mathrm{H}), 4.01(\mathrm{~m}, 4 \mathrm{H}), 3.73(\mathrm{~m}, 4 \mathrm{H}), 1.91$ $(\mathrm{m}, 8 \mathrm{H}), 1.54(\mathrm{~m}, 8 \mathrm{H}), 1.37(\mathrm{~m}, 106 \mathrm{H}), 0.92(\mathrm{t}, 12 \mathrm{H}) .{ }^{13} \mathrm{C} \mathrm{NMR}\left(125 \mathrm{MHz}, \mathrm{CDCl}_{3}\right)$ 146.4, 145.63, $145.58,145.51,144.6,136.4,135.8,125.2,125.1,125.0,123.70,123.68,123.59,115.3,102.7,98.8$, 76.6, 48.5, 46.8, 32.2, 30.9, 30.04, 30.02, 29.98, 29.7, 26.6, 23.0, 19.3, 14.4, 11.8. HRMS calcd for $\mathrm{C}_{132} \mathrm{H}_{174} \mathrm{O}_{4} \mathrm{Si}_{2} \mathrm{Na}[\mathrm{M}+\mathrm{Na}]^{+}$1902.2843, found 1902.2799. 

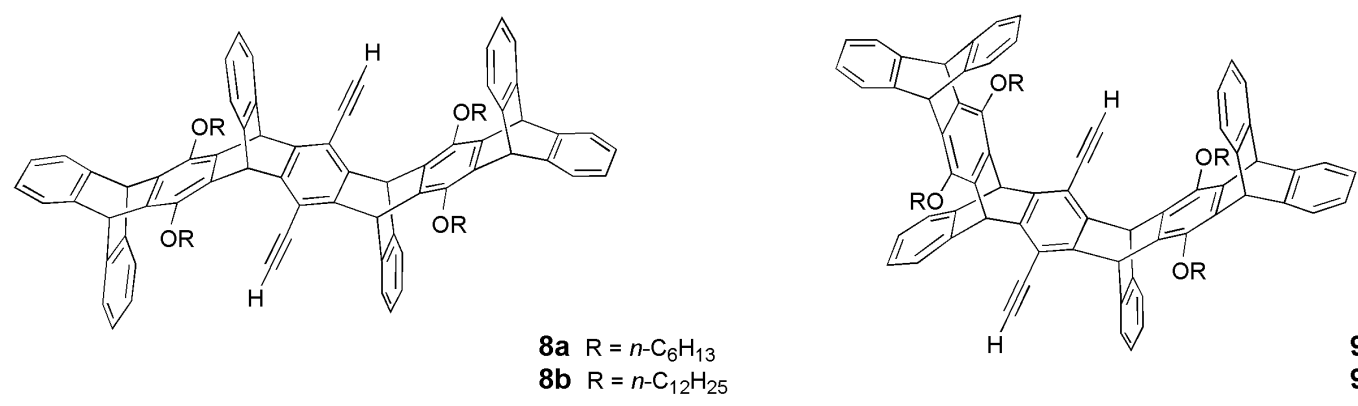

9a $\mathrm{R}=n-\mathrm{C}_{6} \mathrm{H}_{13}$
9b R $\mathrm{R}=n-\mathrm{C}_{12} \mathrm{H}_{25}$

To a solution of $\mathbf{5 c}(0.75 \mathrm{~g}, 0.40 \mathrm{mmol})$ in THF $(10 \mathrm{~mL})$ was added tetrabutylammonium fluoride $(1.0$ M solution in THF, $1.05 \mathrm{~mL}$ ). The reaction mixture was stirred for 3 hours, and the solvent was then removed in vacuo and the crude product was applied to column chromatography and eluted with $\mathrm{CH}_{2} \mathrm{Cl}_{2} / \mathrm{Hexanes}(3: 7, \mathrm{v} / \mathrm{v})$ to give pure product $\mathbf{8 b}$ as a sticky solid $(0.61 \mathrm{~g}, 97 \%)$. 8a and $9 \mathbf{a}-\mathbf{b}$ were prepared using the same procedure. 8a: ${ }^{1} \mathrm{H} \mathrm{NMR}\left(500 \mathrm{MHz}, \mathrm{CDCl}_{3}\right) 7.27(\mathrm{~m}, 4 \mathrm{H}), 7.22(\mathrm{~m}, 8 \mathrm{H}), 6.89$ $(\mathrm{dd}, 4 \mathrm{H}), 6.83(\mathrm{dd}, 4 \mathrm{H}), 6.81(\mathrm{dd}, 4 \mathrm{H}), 6.02(\mathrm{~s}, 4 \mathrm{H}), 5.57(\mathrm{~s}, 4 \mathrm{H}), 3.97(\mathrm{~m}, 4 \mathrm{H}), 3.73(\mathrm{~m}, 4 \mathrm{H}), 3.63(\mathrm{~s}$ 2H), $1.99(\mathrm{~m}, 8 \mathrm{H}), 1.68(\mathrm{~m}, 8 \mathrm{H}), 1.43(\mathrm{~m}, 16 \mathrm{H}), 1.01(\mathrm{t}, 12 \mathrm{H}) .{ }^{13} \mathrm{C} \mathrm{NMR}\left(125 \mathrm{MHz}, \mathrm{CDCl}_{3}\right)$ 146.3, $145.53,145.50,145.1,145.0,136.8,135.7,125.3,125.2,125.1,124.0,123.7,123.6,114.0,84.4,79.5$ 76.5, 48.5, 46.6, 32.1, 30.7, 26.2, 23.0, 14.3. HRMS calcd for $\mathrm{C}_{90} \mathrm{H}_{86} \mathrm{O}_{4} \mathrm{Na}[\mathrm{M}+\mathrm{Na}]^{+} 1253.6424$, found 1253.6553. 9a: ${ }^{1} \mathrm{H}$ NMR (500 MHz, $\left.\mathrm{CDCl}_{3}\right) 7.27$ (m, 8H), $7.21(\mathrm{dd}, 4 \mathrm{H}), 6.90(\mathrm{dd}, 4 \mathrm{H}), 6.85$ (m, 8H), $6.03(\mathrm{~s}, 4 \mathrm{H}), 5.57(\mathrm{~s}, 4 \mathrm{H}), 3.95(\mathrm{~m}, 4 \mathrm{H}), 3.69(\mathrm{~m}, 4 \mathrm{H}), 3.64(\mathrm{~s}, 2 \mathrm{H}), 1.97(\mathrm{~m}, 8 \mathrm{H}), 1.67(\mathrm{~m}, 8 \mathrm{H}), 1.50(\mathrm{~m}$ 16H), $1.04(\mathrm{t}, 12 \mathrm{H}) .{ }^{13} \mathrm{C} \mathrm{NMR}\left(125 \mathrm{MHz}, \mathrm{CDCl}_{3}\right)$ 146.4, 145.7, 145.6, 145.2, 145.1, 136.6, 135.6, $125.4,125.2,125.1,123.9,123.7,123.5,114.0,84.4,79.5,76.5,48.5,46.6,32.2,30.8,26.3,23.0,14.4$. HRMS calcd for $\mathrm{C}_{90} \mathrm{H}_{86} \mathrm{O}_{4} \mathrm{Na}[\mathrm{M}+\mathrm{Na}]^{+}$1253.6424, found 1253.6433. 8b: ${ }^{1} \mathrm{H} \mathrm{NMR}\left(500 \mathrm{MHz}, \mathrm{CDCl}_{3}\right)$ $7.27(\mathrm{~m}, 4 \mathrm{H}), 7.22(\mathrm{~m}, 8 \mathrm{H}), 6.89(\mathrm{dd}, 4 \mathrm{H}), 6.84(\mathrm{dd}, 4 \mathrm{H}), 6.82(\mathrm{dd}, 4 \mathrm{H}), 6.02(\mathrm{~s}, 4 \mathrm{H}), 5.57(\mathrm{~s}, 4 \mathrm{H}), 3.97$ (m, 4H), $3.73(\mathrm{~m}, 4 \mathrm{H}), 3.63(\mathrm{~s}, 2 \mathrm{H}), 1.99(\mathrm{~m}, 8 \mathrm{H}), 1.67(\mathrm{~m}, 8 \mathrm{H}), 1.35(\mathrm{~m}, 64 \mathrm{H}), 0.90(\mathrm{t}, 12 \mathrm{H}) .{ }^{13} \mathrm{C}$ NMR $\left(125 \mathrm{MHz}, \mathrm{CDCl}_{3}\right)$ 146.3, 145.53, 145.49, 145.1, 145.0, 136.8, 135.7, 125.3, 125.23, 125.16, 124.0, $123.7,123.6,114.0,84.5,79.4,76.6,48.5,46.6,32.2,30.8,30.07,30.05,30.02,29.97,29.7,26.6,23.0$, 14.4. HRMS calcd for $\mathrm{C}_{114} \mathrm{H}_{134} \mathrm{O}_{4} \mathrm{Na}[\mathrm{M}+\mathrm{Na}]^{+}$1590.0180, found 1590.0259. 9b: ${ }^{1} \mathrm{H} \mathrm{NMR}(500 \mathrm{MHz}$, 
$\left.\mathrm{CDCl}_{3}\right) 7.26(\mathrm{~m}, 8 \mathrm{H}), 7.20(\mathrm{dd}, 4 \mathrm{H}), 6.89(\mathrm{dd}, 4 \mathrm{H}), 6.84(\mathrm{~m}, 8 \mathrm{H}), 6.02(\mathrm{~s}, 4 \mathrm{H}), 5.56(\mathrm{~s}, 4 \mathrm{H}), 3.94(\mathrm{~m}$, 4H), $3.67(\mathrm{~m}, 4 \mathrm{H}), 3.63(\mathrm{~s}, 2 \mathrm{H}), 1.96(\mathrm{~m}, 8 \mathrm{H}), 1.66(\mathrm{~m}, 8 \mathrm{H}), 1.37(\mathrm{~m}, 64 \mathrm{H}), 0.91(\mathrm{t}, 12 \mathrm{H}) .{ }^{13} \mathrm{C} \mathrm{NMR}$ (125 MHz, $\left.\mathrm{CDCl}_{3}\right)$ 146.4, 145.6, 145.5, 145.11, 145.08, 136.6, 135.6, 125.4, 125.2, 125.1, 124.0, 123.8, 123.5, 114.0, 84.4, 79.4, 76.6, 48.5, 46.5, 32.2, 30.8, 30.14, 30.11, 30.10, 30.07, 30.00, 29.7, 26.7, 23.0, 14.4. HRMS calcd for $\mathrm{C}_{114} \mathrm{H}_{134} \mathrm{O}_{4} \mathrm{Na}[\mathrm{M}+\mathrm{Na}]^{+} 1590.0180$, found 1590.0161 .

Except that 1,4-benzoquinone was used in the place of triptycenequinone, compounds 4a, 5a, 6a and 7 were synthesized via otherwise the same procedures as described above.

4a: ${ }^{1} \mathrm{H}$ NMR (500 MHz, $\left.\mathrm{CDCl}_{3}\right) 8.88(\mathrm{~s}, 2 \mathrm{H}), 7.96(\mathrm{~m}, 2 \mathrm{H}), 7.46$ (m, 4H), 7.07 (m, 2H), $6.67(\mathrm{~s}, 2 \mathrm{H})$, $6.57(\mathrm{~s}, 2 \mathrm{H}), 4.05(\mathrm{~m}, 2 \mathrm{H}), 3.95(\mathrm{~m}, 2 \mathrm{H}), 1.84(\mathrm{~m}, 4 \mathrm{H}), 1.38(\mathrm{~m}, 54 \mathrm{H}), 0.92(\mathrm{t}, 6 \mathrm{H}) .{ }^{13} \mathrm{C}$ NMR $(125$ $\left.\mathrm{MHz}, \mathrm{CDCl}_{3}\right) 148.4,144.6,144.5,133.8,132.0,129.5,128.3,125.6,125.53,125.51,124.2,115.6$, $110.9,102.8,100.8,69.5,45.8,31.7,29.3,25.7,22.7,19.00,18.97,14.1,11.6$. HRMS calcd for $\mathrm{C}_{62} \mathrm{H}_{82} \mathrm{O}_{2} \mathrm{Si}_{2} \mathrm{Na}[\mathrm{M}+\mathrm{Na}]^{+}$937.5746, found 937.5707.

5a: ${ }^{1} \mathrm{H}$ NMR (500 MHz, $\left.\mathrm{CDCl}_{3}\right) 7.27$ (dd, 4H), 6.91 (dd, 4H), 6.44 (s, 4H), 6.36 (s, 4H), 3.97 (m, 4H), $3.88(\mathrm{~m}, 4 \mathrm{H}), 1.73(\mathrm{~m}, 8 \mathrm{H}), 1.37(\mathrm{~m}, 66 \mathrm{H}), 0.90(\mathrm{t}, 12 \mathrm{H}) .{ }^{13} \mathrm{C} \mathrm{NMR}\left(125 \mathrm{MHz}, \mathrm{CDCl}_{3}\right)$ 148.9, 146.8, 145.2, 135.9, 125.5, 124.4, 116.1, 111.3, 103.1, 98.5, 70.1, 46.5, 32.4, 29.9, 26.4, 23.4, 19.73, 19.69, 14.8, 12.4. HRMS calcd for $\mathrm{C}_{80} \mathrm{H}_{110} \mathrm{O}_{4} \mathrm{Si}_{2} \mathrm{Na}[\mathrm{M}+\mathrm{Na}]^{+} 1213.7835$, found 1213.7863 . 6a: ${ }^{1} \mathrm{H}$ NMR (500 $\left.\mathrm{MHz}, \mathrm{CDCl}_{3}\right) 7.28(\mathrm{dd}, 4 \mathrm{H}), 6.91(\mathrm{dd}, 4 \mathrm{H}), 6.42(\mathrm{~s}, 4 \mathrm{H}), 6.38(\mathrm{~s}, 4 \mathrm{H}), 3.93(\mathrm{~m}, 4 \mathrm{H}), 3.84(\mathrm{~m}, 4 \mathrm{H}), 1.78$ (m, 8H), $1.37(\mathrm{~m}, 66 \mathrm{H}), 0.91(\mathrm{t}, 12 \mathrm{H}) .{ }^{13} \mathrm{C} \mathrm{NMR}\left(125 \mathrm{MHz}, \mathrm{CDCl}_{3}\right)$ 149.0, 146.6, 145.3, 135.8, 125.5, 124.4, 116.1, 110.9, 103.4, 98.5, 70.0, 46.4, 32.4, 30.1, 26.4, 23.4, 19.8, 14.8, 12.3. HRMS calcd for $\mathrm{C}_{80} \mathrm{H}_{110} \mathrm{O}_{4} \mathrm{Si}_{2} \mathrm{Na}[\mathrm{M}+\mathrm{Na}]^{+} 1213.7835$, found 1213.7862 .

Two stereoisomers of 7 give almost identical spectra: ${ }^{1} \mathrm{H}$ NMR $\left(500 \mathrm{MHz}, \mathrm{CDCl}_{3}\right) 7.36(\mathrm{dd}, 4 \mathrm{H}), 6.92$ (dd, 4H), $6.43(\mathrm{~s}, 4 \mathrm{H}), 6.29(\mathrm{~s}, 4 \mathrm{H}), 3.87(\mathrm{t}, 8 \mathrm{H}), 3.61(\mathrm{~s}, 2 \mathrm{H}), 1.81(\mathrm{q}, 8 \mathrm{H}), 1.56(\mathrm{~m}, 8 \mathrm{H}), 1.39(\mathrm{~m}, 16 \mathrm{H})$, 0.95 (t, 12H). ${ }^{13} \mathrm{C} \mathrm{NMR}\left(125 \mathrm{MHz}, \mathrm{CDCl}_{3}\right)$ 148.8, 145.5, 145.2, 135.4, 125.2, 124.1, 114.5, 111.2, 84.1, 
79.4, 70.0, 45.8, 32.0, 29.8, 26.1, 22.9, 14.4. HRMS calcd for $\mathrm{C}_{62} \mathrm{H}_{70} \mathrm{O}_{4} \mathrm{Na}[\mathrm{M}+\mathrm{Na}]^{+}$901.5172, found 901.4973.
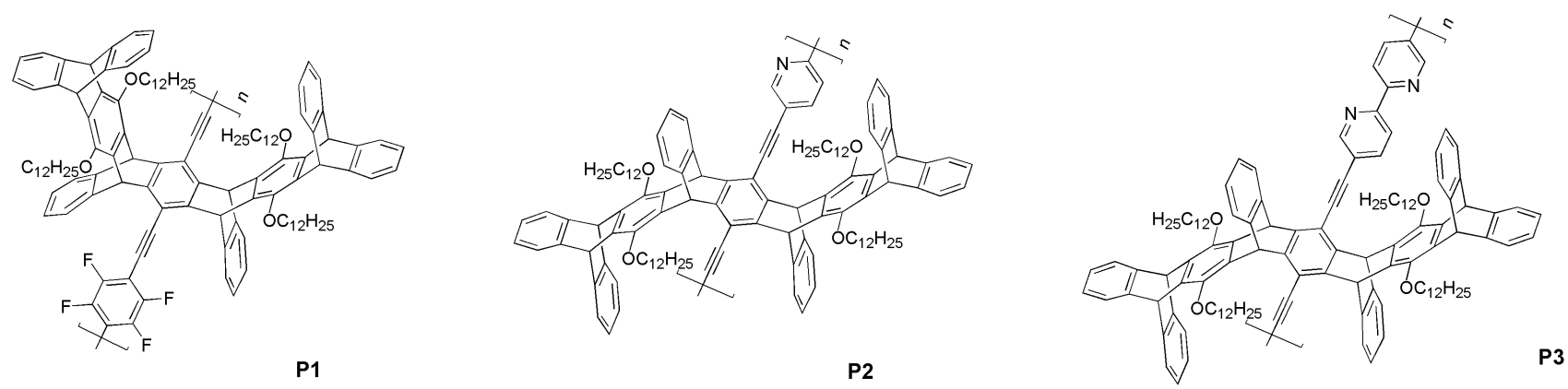

General Procedure for Polymer Syntheses. A general procedure is illustrated by the synthesis of $\mathbf{P 1}$. 9b (30 mg, $0.02 \mathrm{mmol}), 1,4$-diiodotetrafluorobenzene (7.5 mg, $0.187 \mathrm{mmol}), \mathrm{CuI}(6 \mathrm{mg})$, and $\mathrm{Pd}\left(\mathrm{PPh}_{3}\right)_{4}$ $(8 \mathrm{mg})$ were placed in a Schlenk tube with a stir bar. The flask was evacuated and back-filled with argon three times before the addition of degassed toluene/diisopropylamine $(7: 3, \mathrm{v} / \mathrm{v}, 2.5 \mathrm{~mL})$ under an argon atmosphere. The tube was then sealed and heated at $60{ }^{\circ} \mathrm{C}$ for 3 days and subject to a $\mathrm{CH}_{3} \mathrm{Cl} / \mathrm{H}_{2} \mathrm{O}$ workup. The combined organic layer was washed with sat. $\mathrm{NH}_{4} \mathrm{Cl}$ twice followed by brine, and then dried over anhydrous $\mathrm{Na}_{2} \mathrm{SO}_{4}$. The solvent was removed in vacuo. The residue was dissolved in a minimum amount of chloroform and reprecipitated in methanol. The precipitate was washed with hot methanol and acetone. The procedure was repeated three times to give $\mathbf{P 1}$ as a yellow solid (45\%): ${ }^{1} \mathrm{H}$ NMR (300 MHz, $\left.\mathrm{CDCl}_{3}\right)$ : 7.31 (br, 12H), $6.93(\mathrm{br}, 12 \mathrm{H}), 6.26$ (br, 4H), $5.25(\mathrm{br}, 4 \mathrm{H}), 3.98(\mathrm{br}, 8 \mathrm{H})$, $2.05(\mathrm{br}, 8 \mathrm{H}), 1.2(\mathrm{br}, 84 \mathrm{H})$.

P2 and P3 were synthesized using the similar procedure for P1. P2 (86 \%): ${ }^{1} \mathrm{H}$ NMR (500 MHz, $\mathrm{CDCl}_{3}$ ): $9.28(\mathrm{br}, 1 \mathrm{H}), 8.07(\mathrm{br}, 1 \mathrm{H}), 8.01(\mathrm{br}, 1 \mathrm{H}), 7.37(\mathrm{br}, 12 \mathrm{H}), 6.95(\mathrm{br}, 12 \mathrm{H}), 6.30(\mathrm{br}, 4 \mathrm{H}), 5.71$ (br, 4H), 4.05 (br, 8H), 2.09 (br, 8H), 1.2 (br, 72H), 1.05 (br, 12H). P3 (79 \%): ${ }^{1} \mathrm{H}$ NMR (500 MHz, $\mathrm{CDCl}_{3}$ ): $9.24(\mathrm{br}, 2 \mathrm{H}), 8.84(\mathrm{br}, 2 \mathrm{H}), 8.31$ (br, 2H), 7.33 (br, 12H), 6.93 (br, 12H), $6.22(\mathrm{br}, 4 \mathrm{H}), 5.65$ (br, 4H), 3.95 (br, 8H), 1.99 (br, 8H), 1.57 (br, 16H) 1.3 (br, 56H), 0.82 (br, 12H). 


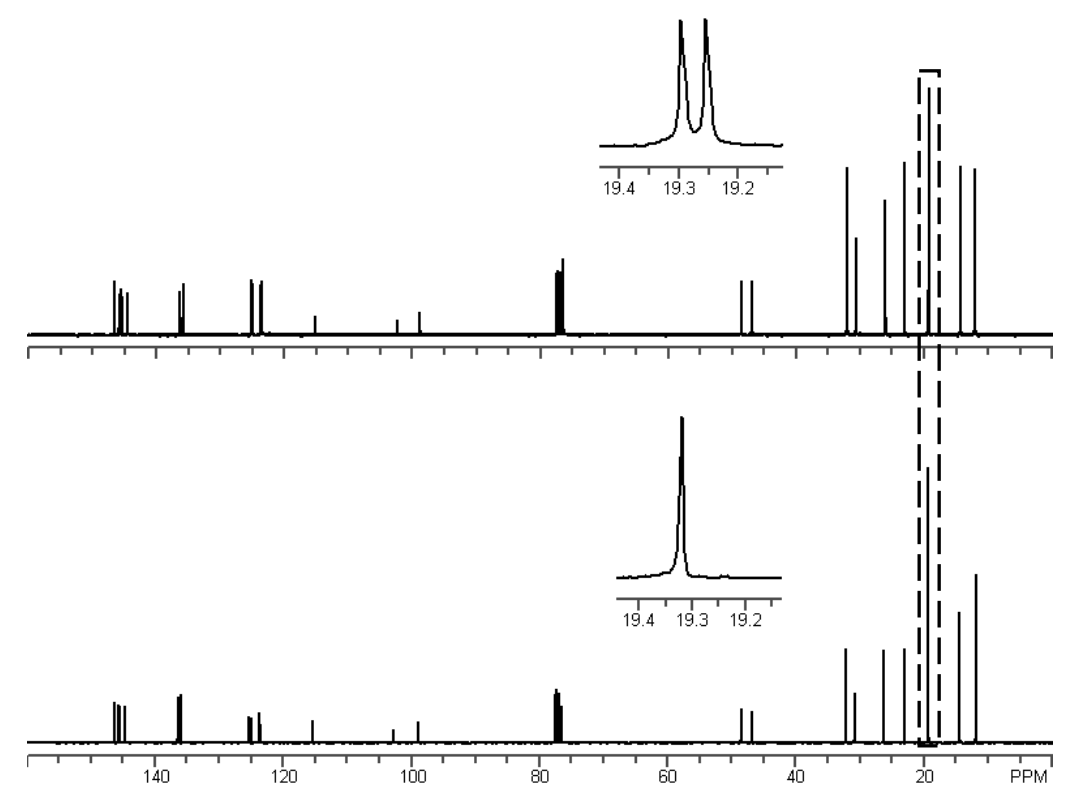

Figure S1. ${ }^{13} \mathrm{C}$ NMR spectra of $\mathbf{5 b}$ (top) and $\mathbf{6 b}$ (bottom). The insets show the expanded images of the highlighted peaks in the dashed box.
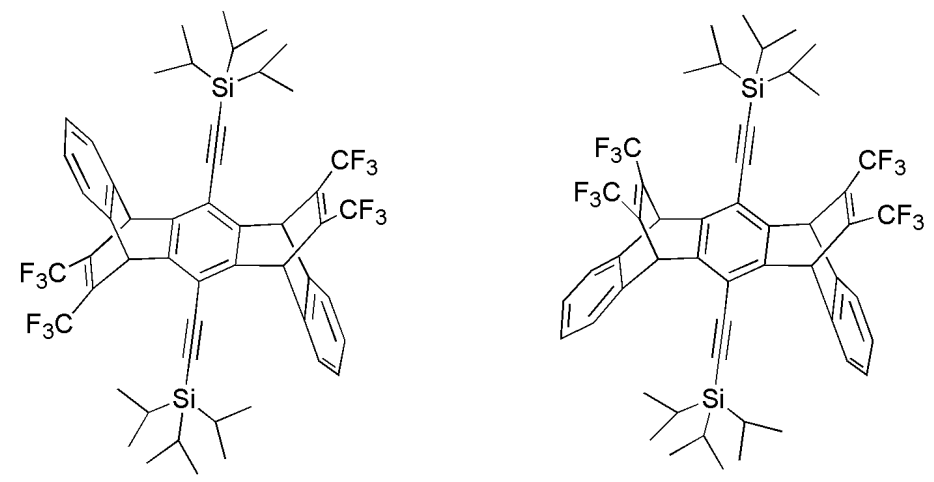

Chart S1. Chemical structures of related isomers, which exhibited similar signal splitting as 5 and 6 (Figure S1) in the carbons of the methyl group in TIPS unit. The single crystal structure of these compounds will be independently published. ${ }^{3}$

\section{References}

(1) Anthony, J. E.; Brooks, J. S.; Eaton, D. L.; Parkin, S. R. J. Am. Chem. Soc. 2001, 123, 9482-9483.

(2) Williams, V. E.; Swager, T. M. Macromolecules 2000, 33, 4069-4073.

(3) Kim, Y.; Whitten, J. E.; Swager, T. M., submitted. 
${ }^{1} \mathrm{H}$ and ${ }^{13} \mathrm{C}$ NMR spectra of $\mathbf{4 b}$
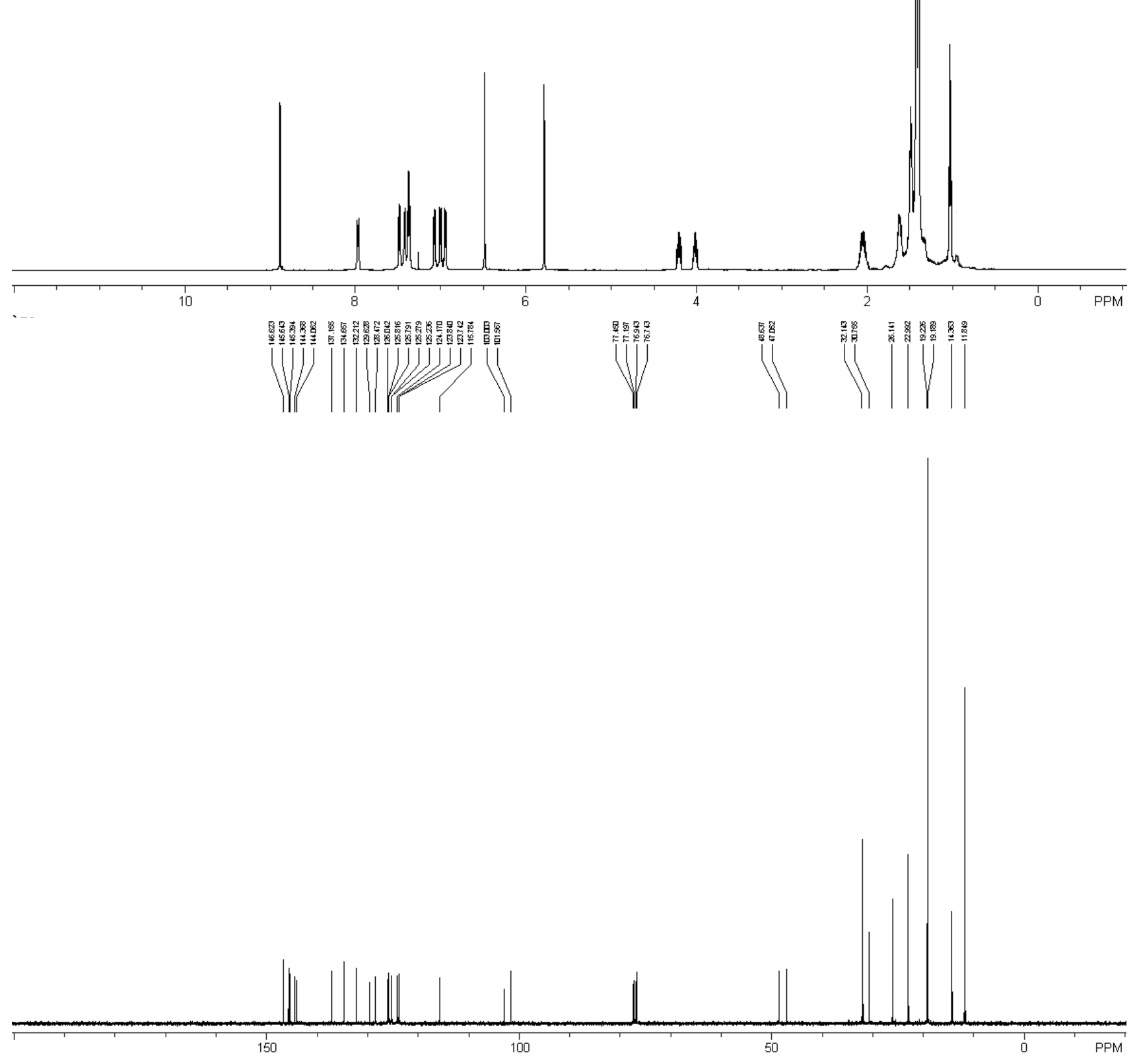
${ }^{1} \mathrm{H}$ and ${ }^{13} \mathrm{C}$ NMR spectra of $\mathbf{4 c}$

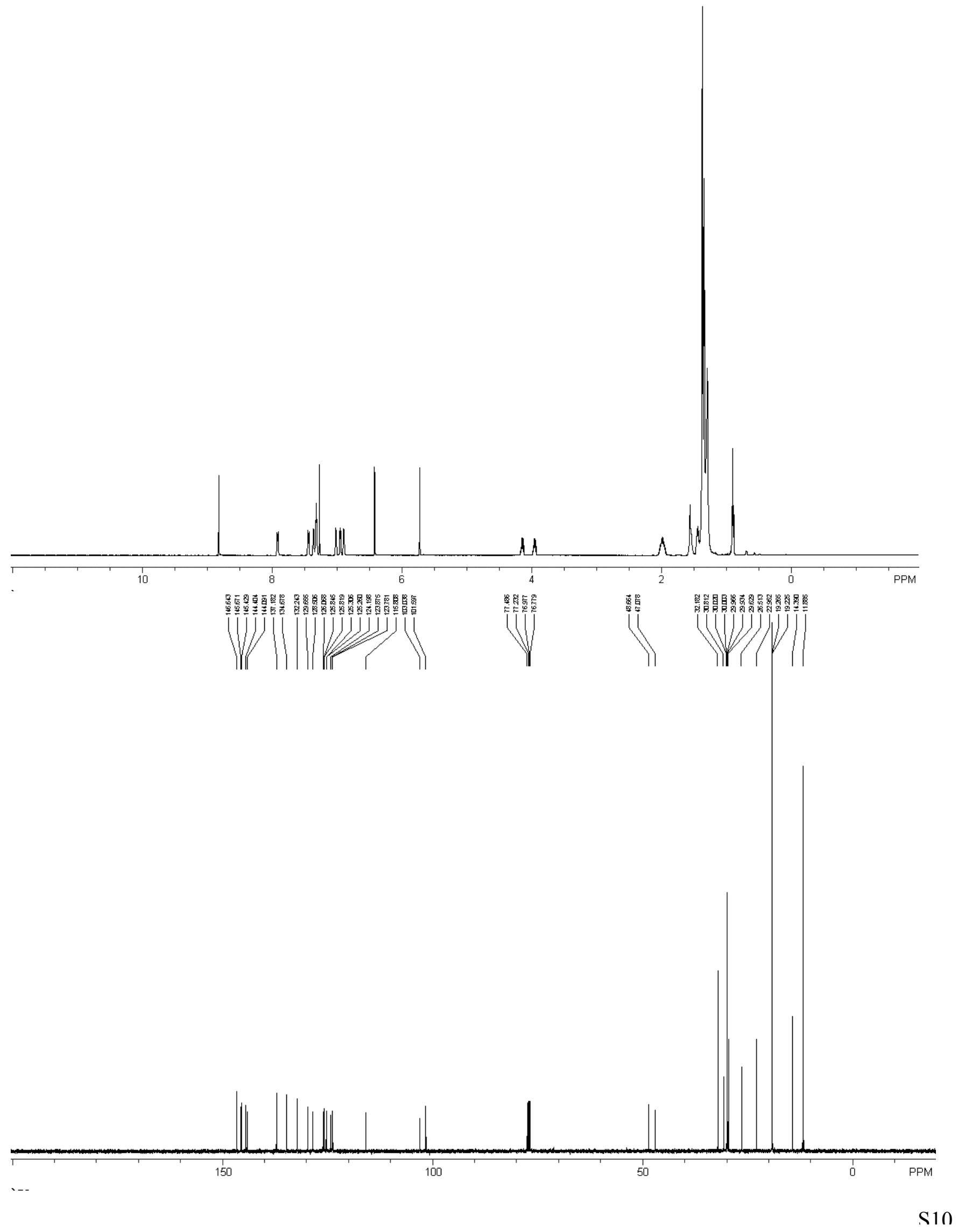


${ }^{1} \mathrm{H}$ and ${ }^{13} \mathrm{C}$ NMR spectra of $\mathbf{5 b}$
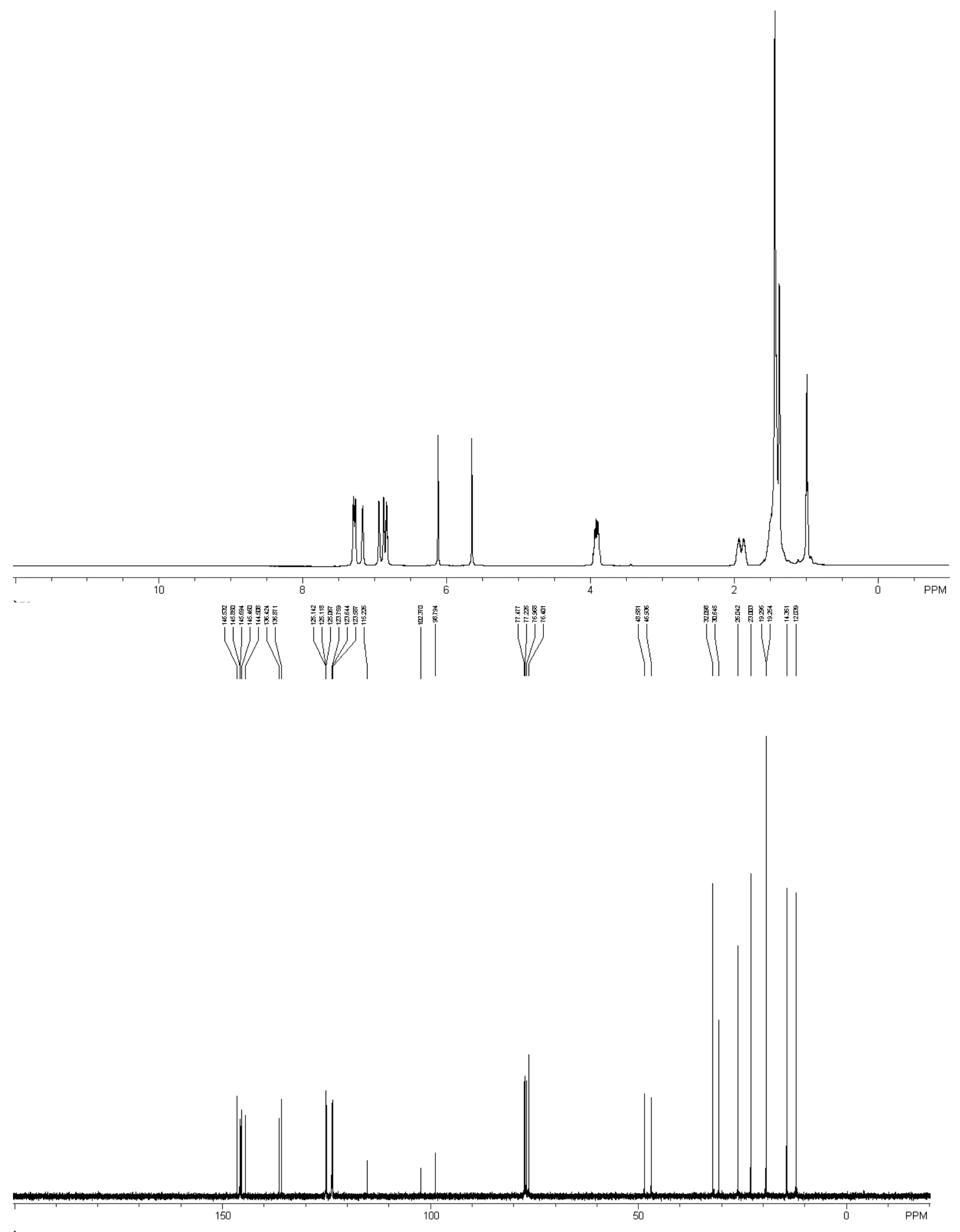
${ }^{1} \mathrm{H}$ and ${ }^{13} \mathrm{C}$ NMR spectra of $\mathbf{5 c}$

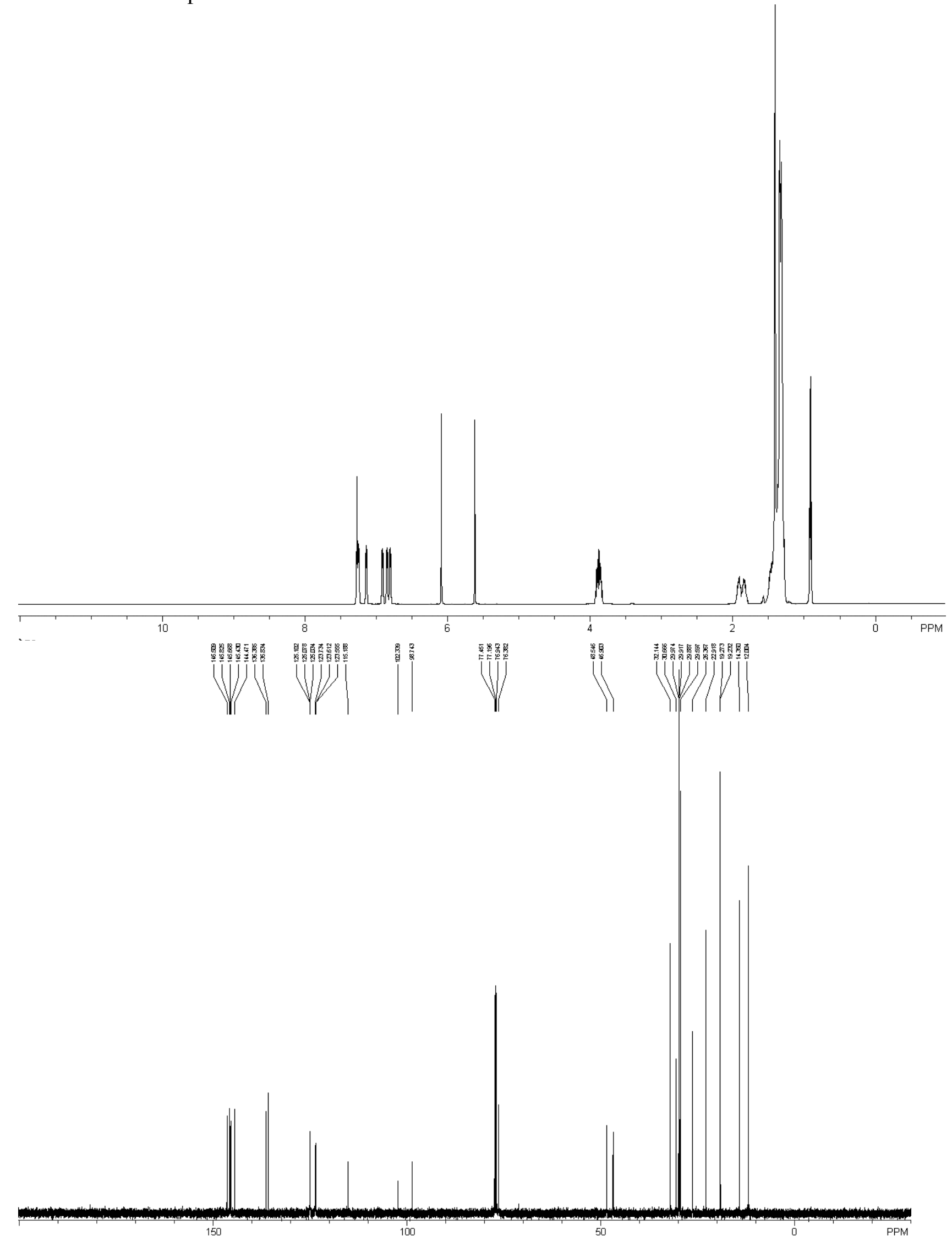


${ }^{1} \mathrm{H}$ and ${ }^{13} \mathrm{C}$ NMR spectra of $\mathbf{6 b}$
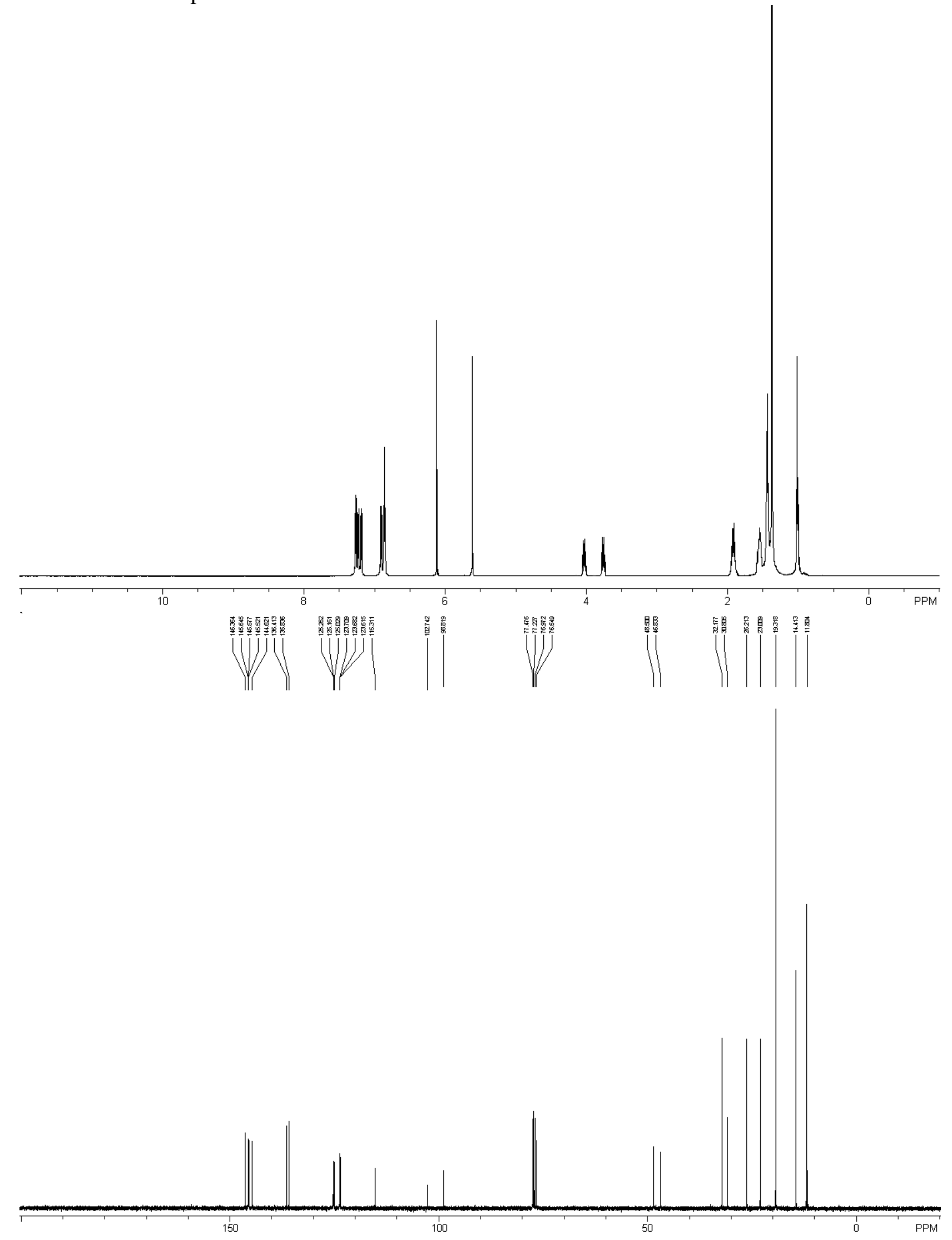
${ }^{1} \mathrm{H}$ and ${ }^{13} \mathrm{C}$ NMR spectra of $\mathbf{6 c}$

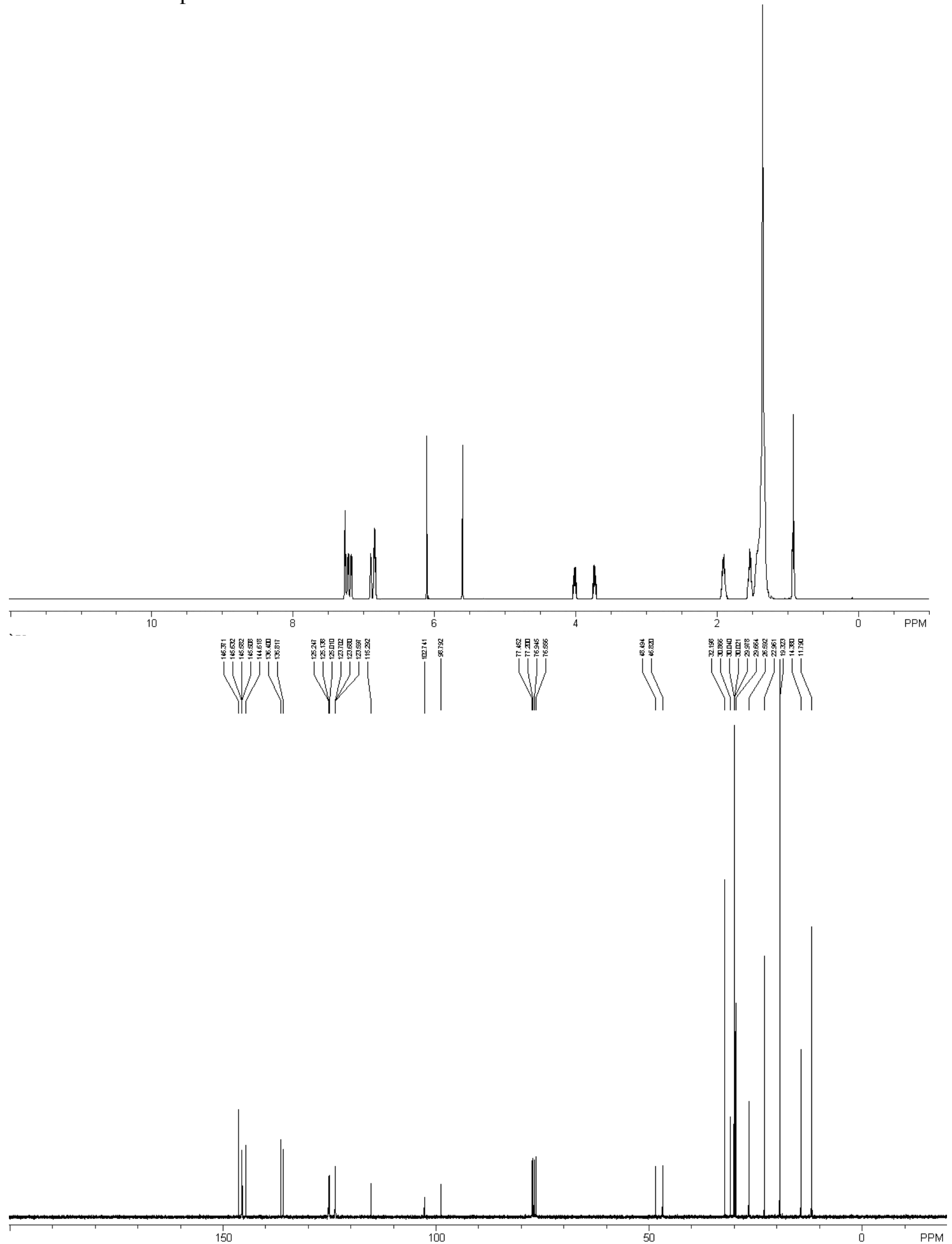


${ }^{1} \mathrm{H}$ spectra of 7

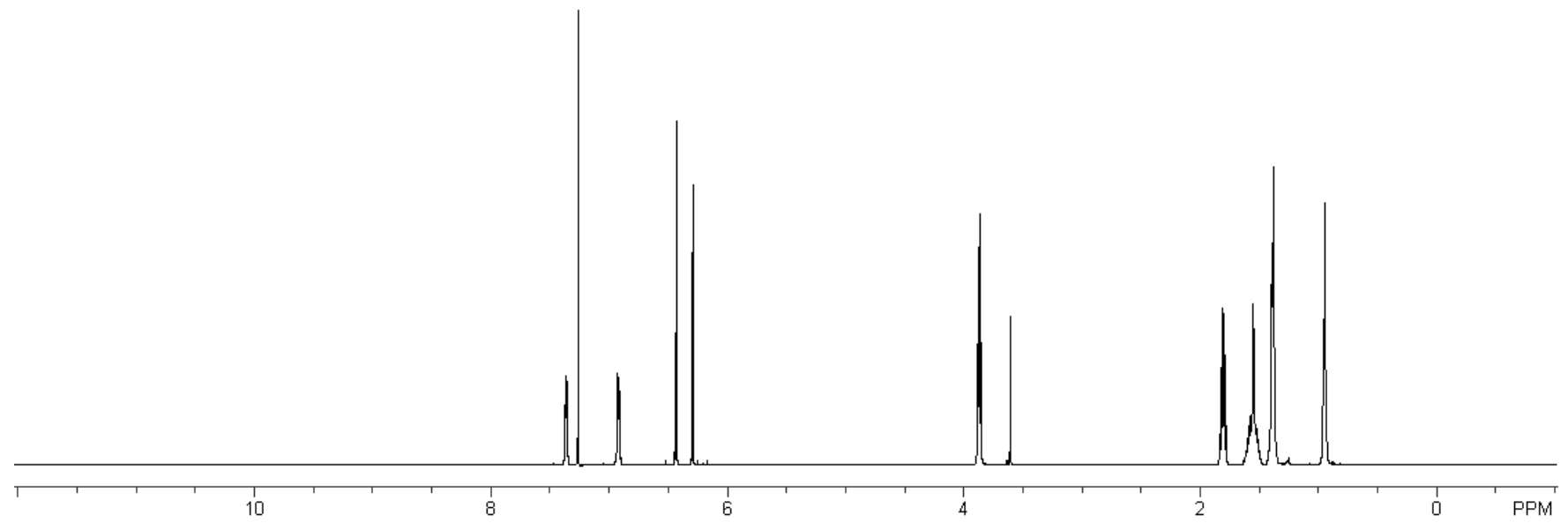

${ }^{1} \mathrm{H}$ spectra of $\mathbf{8 a}$

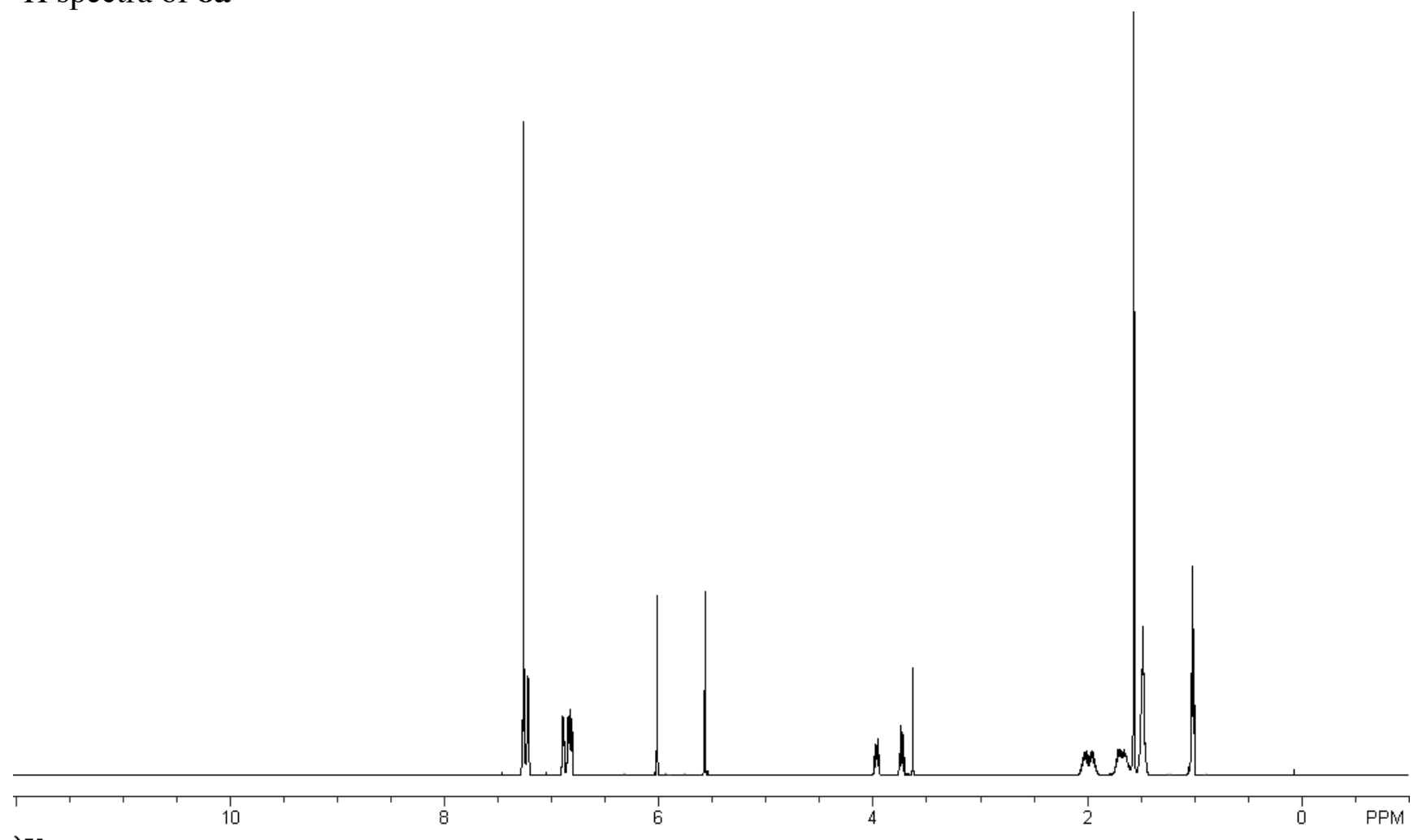


${ }^{1} \mathrm{H}$ and ${ }^{13} \mathrm{C}$ NMR spectra of $\mathbf{8 b}$
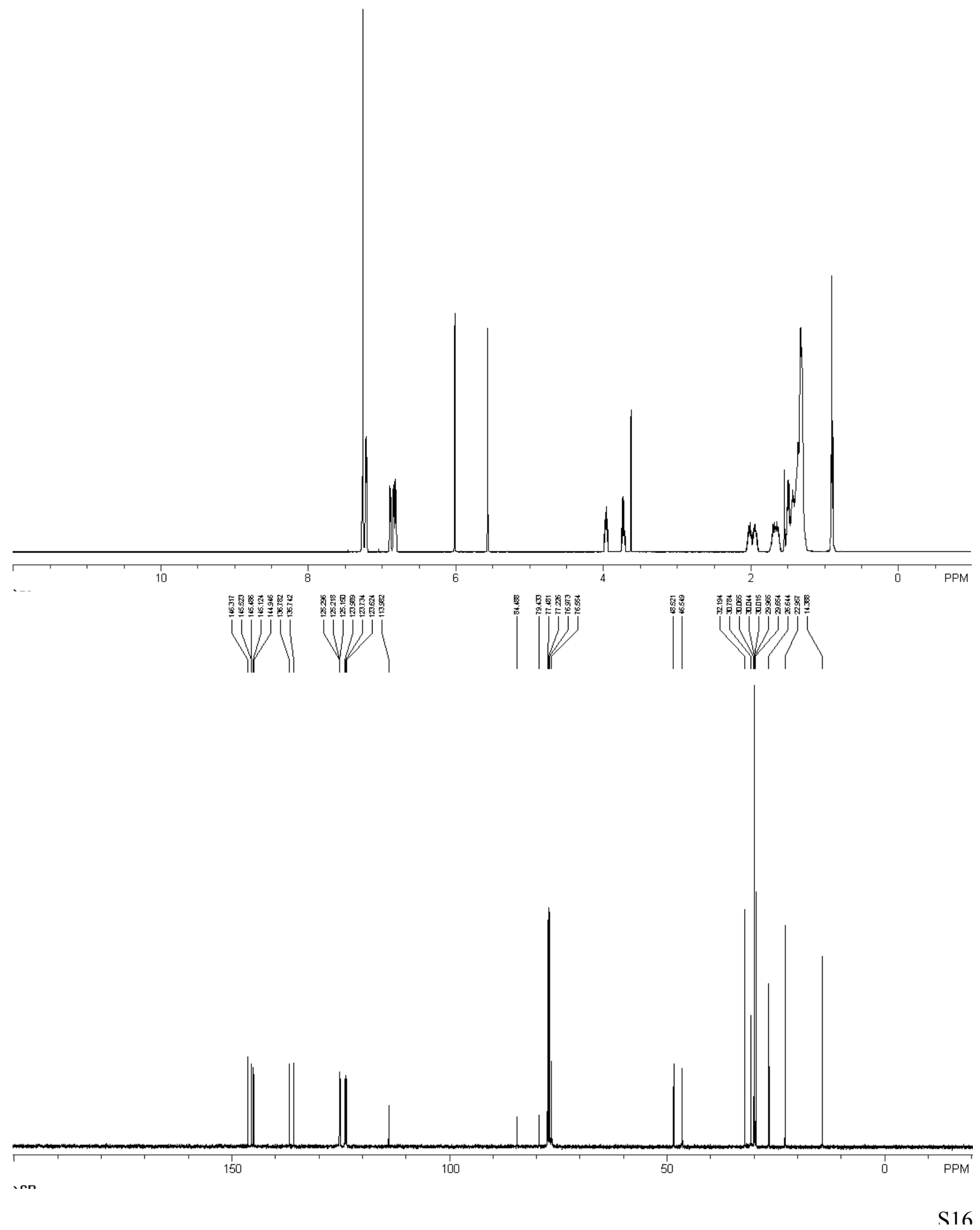
${ }^{1} \mathrm{H}$ and ${ }^{13} \mathrm{C}$ NMR spectra of 9a
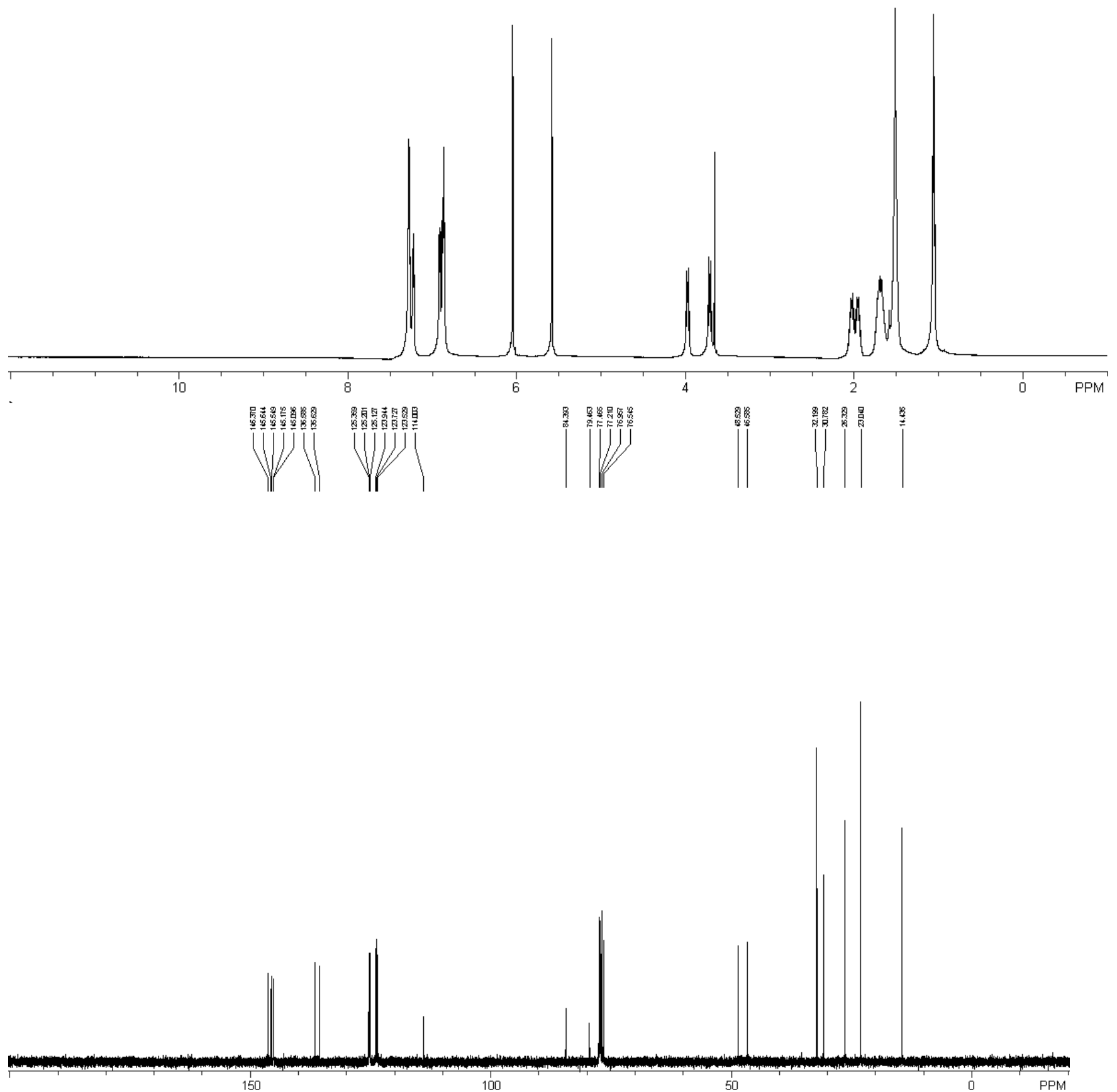
${ }^{1} \mathrm{H}$ and ${ }^{13} \mathrm{C}$ NMR spectra of $\mathbf{9 b}$
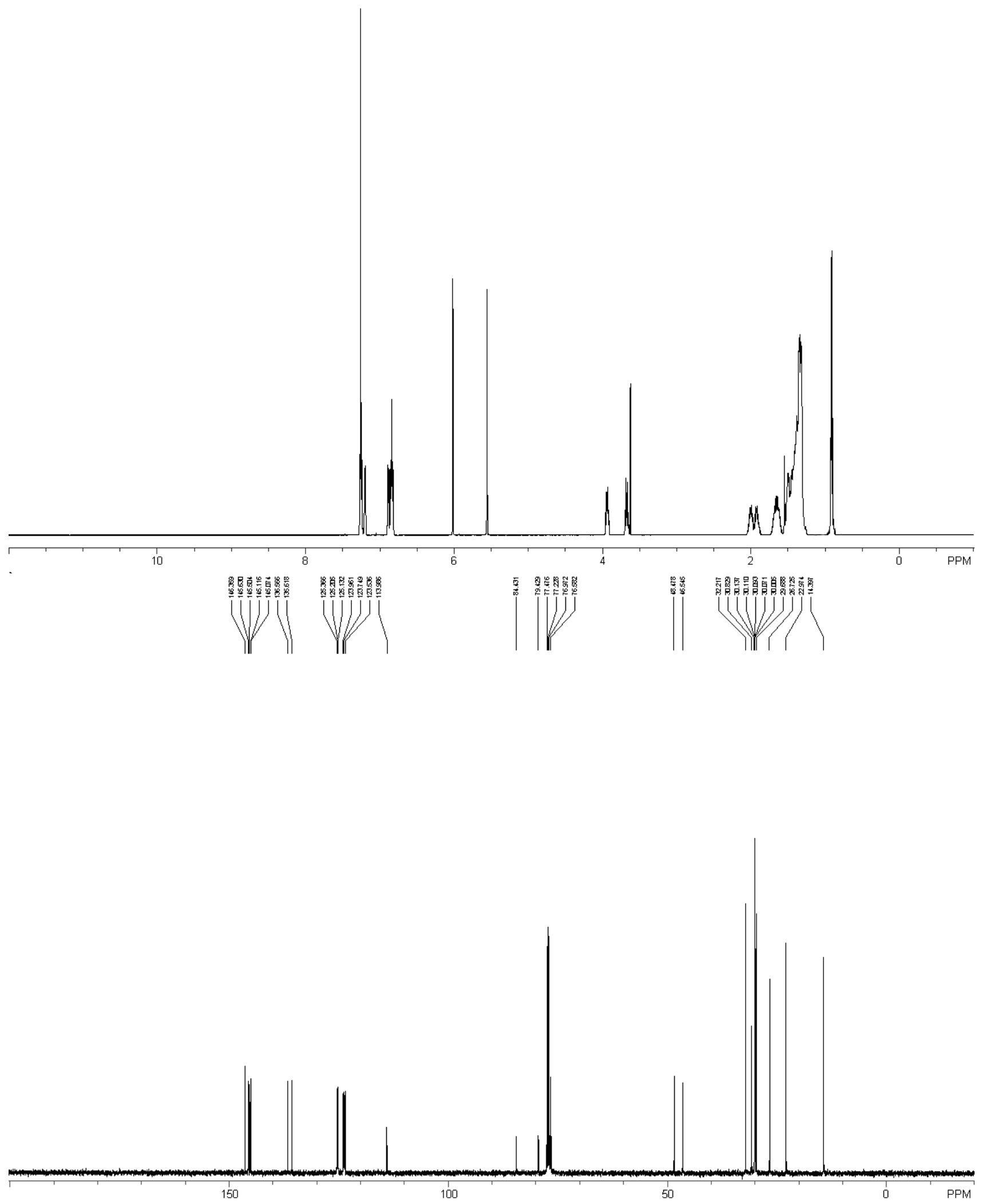
${ }^{1} \mathrm{H}$ and ${ }^{13} \mathrm{C}$ NMR spectra of $\mathbf{4 a}$

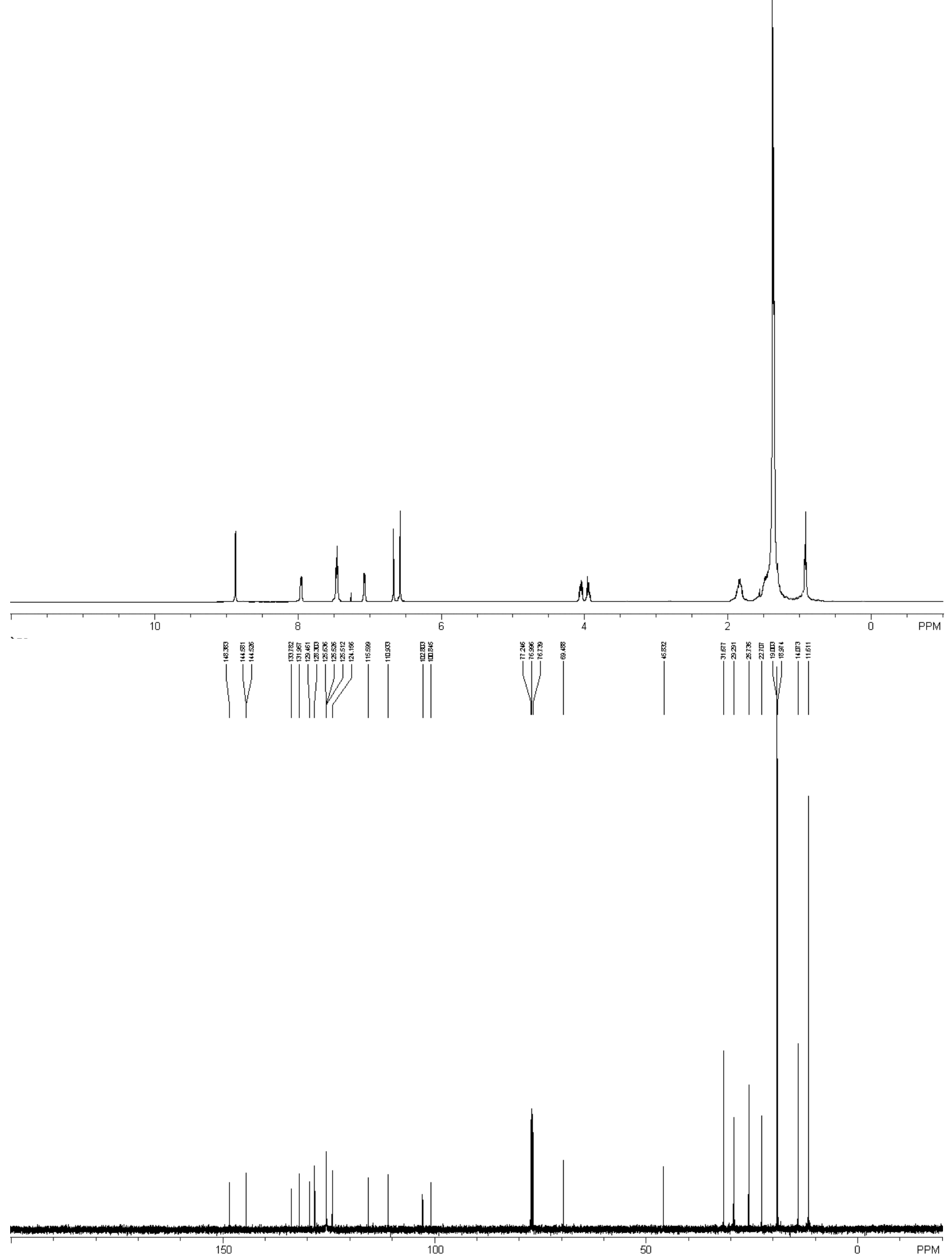


${ }^{1} \mathrm{H}$ and ${ }^{13} \mathrm{C}$ NMR spectra of $\mathbf{5 a}$

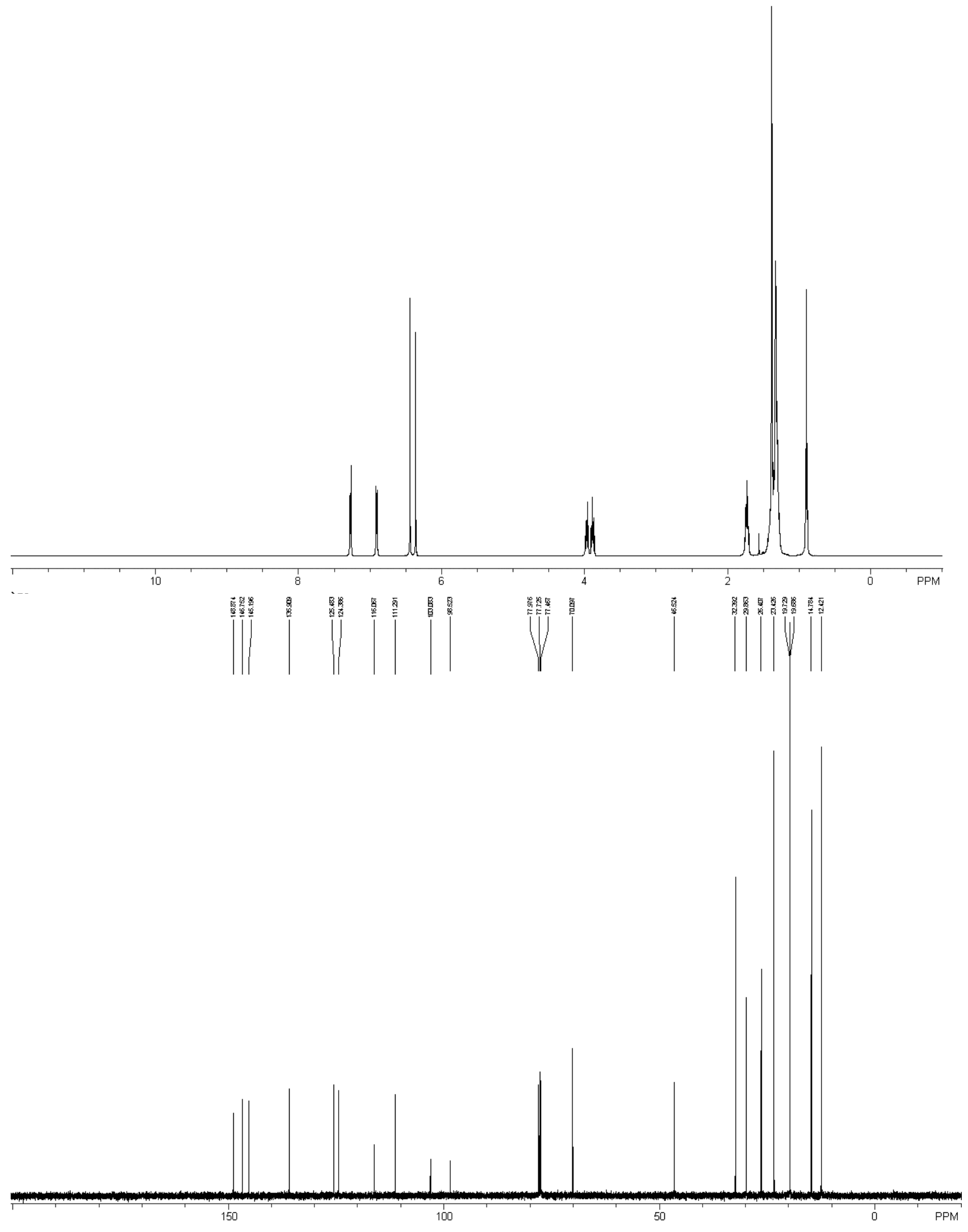


${ }^{1} \mathrm{H}$ and ${ }^{13} \mathrm{C}$ NMR spectra of $\mathbf{6 a}$
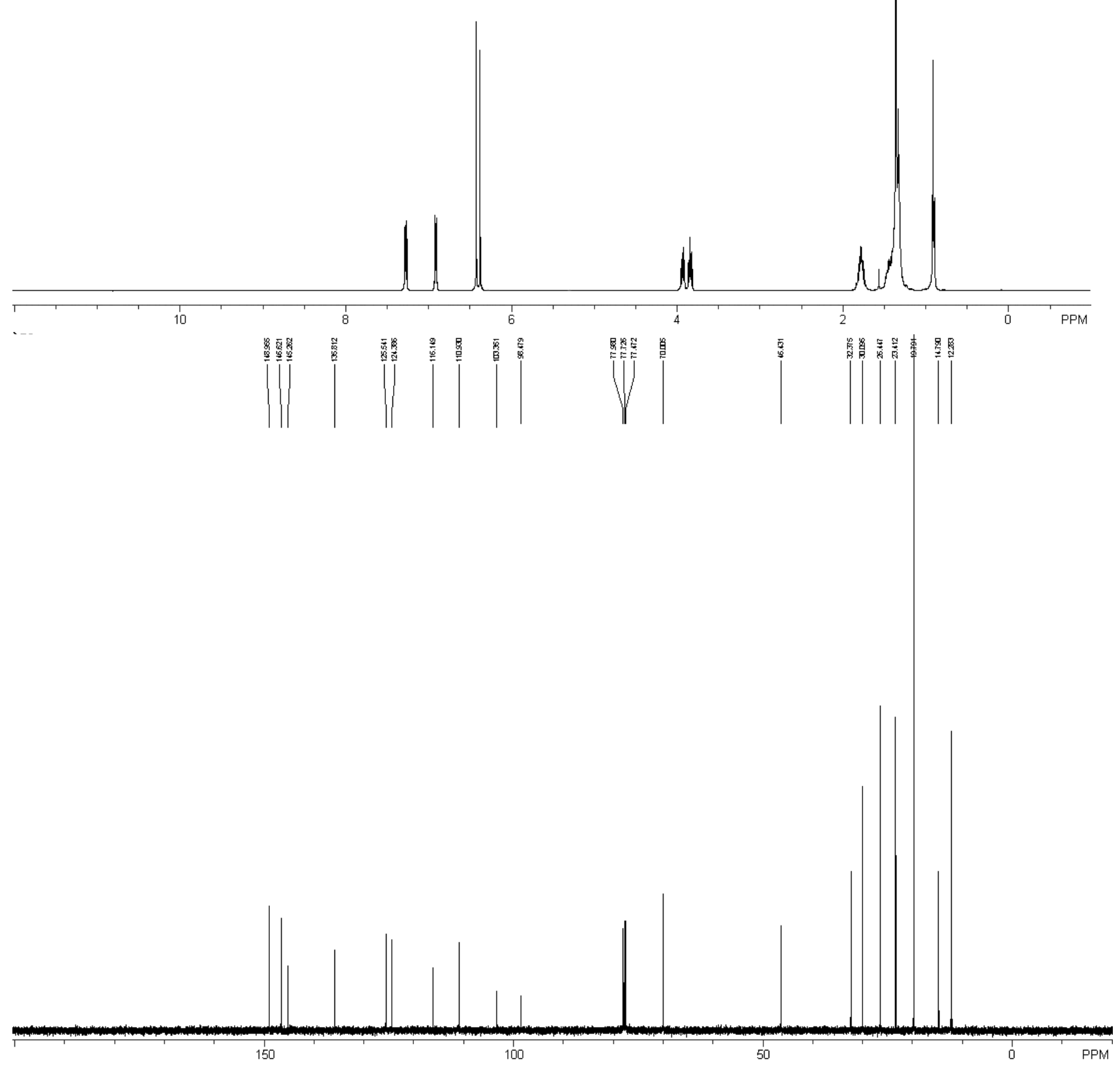